\title{
Measurement Instruments and Software Used in Biotribology Research Laboratory
}

\author{
Andrei Tyurin (Researcher, National Research University of Information Technologies, Mechanics and Optics - \\ ITMO), German Ganus (Junior Researcher, ITMO), Mikhail Stepanov (Junior Researcher, ITMO), \\ Gafurzhan Ismailov (Associate Professor, Tomsk State Pedagogical University)
}

\begin{abstract}
Precision measurements of friction processes have a key role in a variety of industrial processes. The emergence of fine electronic circuit techniques greatly expands capabilities of control. There are some difficulties for their full implementation today, especially when it regards the accuracy and frequency of measurements. The motion-measuring method in real-time system is considered in this article, paying special attention to increased accuracy. This method is based on rapid analog digital converter (ADC), transmission program and digital signal processor (DSP) algorithms. Description of laboratory devices is included: Tribal-T and universal friction machine (MTU-01) designed for "Pin on disc" tests. Great emphasis is placed on the usability of accelerometers. The present study examined the collected data via laboratory system for data acquisition and control, and processing it in the laboratory of Biotribology. Laboratory supervisory control and data acquisition (SCADA) algorithms is described below. Task of regulation is not considered. This paper describes only methods of automatic control theory to analyze the frictional quality.
\end{abstract}

Keywords - Friction; Mixed analog digital integrated circuits; Frequency-domain analysis; Real-time systems; WEB services.

\section{INTRODUCTION}

Measurement of object position, displacement of its various mechanisms, details, parts, products etc. is one of engineering goals. Some measurements constitute the synthesis of contact method. The object's movement under conditions of dry friction is considered in this study. The subject being examined is the friction interactions of surfaces of two details of different materials.

Friction has many properties and effects, the main of which is nonlinear movement under the conditions of friction. To develop the original device Tribal-T special equipment was by the researchers of theDepartment of Mechatronics of ITMO University in St. Petersburg. "Tribal-T" was designed to determine friction parameters according to the input-output model: coefficient of dynamic friction, natural frequency, damping factor. The input-output dynamic model is based on measured data. These data present the replacement of upper and lower mobile platforms, (Fig. 1) show schematics of device. Tribological properties of materials were researched in agreement with the device. The device consists of the mechanical, control, contact and non-contact measuring systems.

a. The mechanical system includes the basis, linear guide for mobile platforms with sample-holders, upper and lower samples "1". Upper and lower samples generate friction pair. Demountable sample-holders extend a range of testing elements. Electric alternating current (AC) motor "8" sets in back-and-forth motion the down platform by the crank gear. The upper platform is fixed by two springs; that way the upper platform is set in back-and-forth motion by the sample's contact " 1 ". Reversive AC motor pressures the samples by worm gear " 6 ".

b. The contact measuring system is necessary to define mechanical characteristics of samples. This system consists of linear variable differential transformer (LVDT) " 2 " and resistance strain load sensor " 3 ". LVDT measures the replacement of upper and lower platforms. A load sensor is placed between the worm gear and upper linear guide, which allows to measure the load force between the samples.

c. The control system allows operating of electric AC motor device " 6 ", transforming and processing the data from measuring system. The control system operates the device " 7 " and consists of ADC/DAC module, digital input/output, and the special PC software from "L-Card" company.

The control system has the following functional characteristics:

- USB interface;

- DSP ADSP-2185M with a clock speed of $48 \mathrm{MHz}$;

- 16 differential channels or 32 channels with common ground for analog input with automatic adjustment of the data;

- the maximum frequency of the ADC is $400 \mathrm{kHz}$;

- two inputs for external digital sync input analog signals;

- DAC with two analog output channels with maximum summation frequency $125 \mathrm{kHz}$.

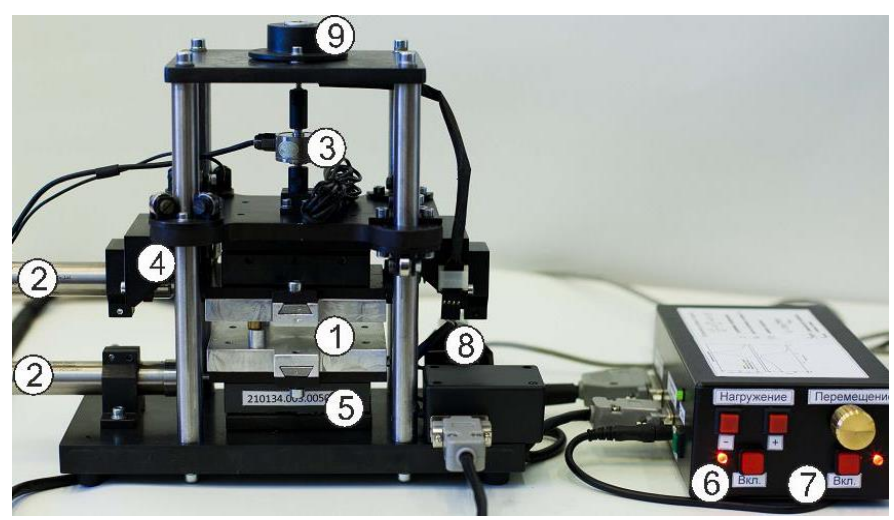

Fig. 1. The schematics of the device "Tribal-T" 
The device "Tribal-T" has the following technical characteristics:

- The type of moving back-and-forth (plane-plane); tests can be taken with and without lubricant;

- Displacement range: 0.1-5.0 mm;

- Displacement frequency range: 0.1-5.0 Hz;

- Speed of loading: $2.5 \mathrm{~mm} / \mathrm{s}$;

- Max. range of loading system: $33.0 \mathrm{~mm}$;

- Power voltage 220 V, $50 \mathrm{~Hz}$;

- Dimensions, cm: 198x121x181;

- Load force: 2-50 N, precision $4 \%$.

Description of the device work: movement is transferred from the engine " 8 " through the slide-crank mechanism to the bottom platform with holders, one of which, in turn, actuates samples. Vertical load on samples is created by the screw jack 6 set in motion by the reversive engine through a worm gear. The load is transferred through the strain gauge " 3 ", its indications are inserted into the computer through ADC module and digital input/output "7".

In this work relative moving of platforms with samples is estimated. Movement tracing is tracked by contact measures.

\section{FEATURES OF THE PROCESS}

The movement of two platforms is the investigated process of this work. It appears to us in the form of the relative motion of two linear guides on the process of constantly acting friction forces that arise and then again are approaching zero. We describe the process in more detail; slide-crank rod mechanism in motion is represented as vectors of force and acceleration. At the beginning there is no speed, only acceleration and then there is a transition at the midpoint of the rate it increases reaching the maximum and the acceleration drops to zero. The behavior of the upper platform is similar to the bottom one, but in addition it is affected by the friction force on the plane.

The plane of the spring is attached to the upper platform. At the beginning of the cycle the spring is compressed until the breakdown and subsequent decompression transfer energy to the surge. In Touch is a scheme in the plane of the plane is used by three of the bottom three and balanced them on top of the contact area is much smaller than the actual contact area, mainly due to topography.

\section{MODIFIED PIN ON DISC TRIBOMETER}

The equipment for tribological "Pin on disc" tests was specifically designed to find dynamic parameters of friction contact. Tribometer allows to measure linear displacement of input " 6 " and output " 5 " under dynamics of friction process [2]. Based on the calculated data the dynamic model of tribocontact traces the evolution of the parameters over time.

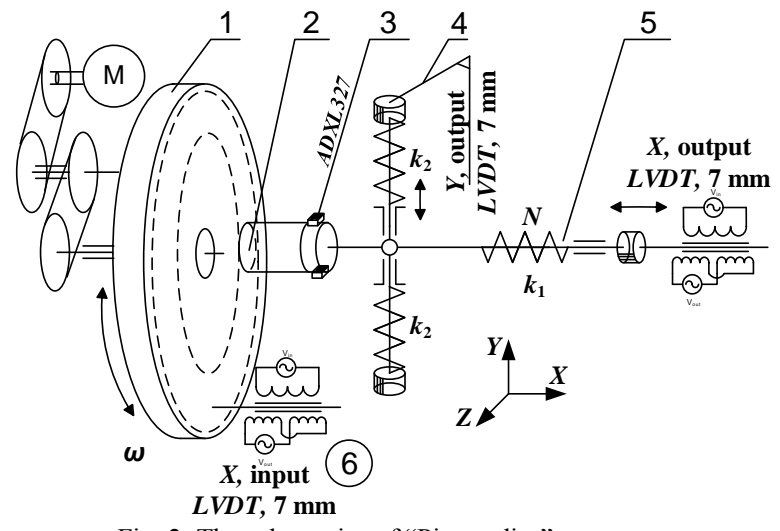

Fig. 2. The schematics of "Pin on disc" test systems.

An instrument for experimental research was created to test its schematic diagram (Fig. 2). In the mechanical part to implement the scheme contact the indenter " 2 " on disk " 1 ". The drive unit consists of an asynchronous motor " $M$ " driving the rotation disc through the serial transfer belt " 4 ".

TABLE I

CHARACTERISTICS OF USED CHANNELS

\begin{tabular}{|c|c|c|c|}
\hline $\begin{array}{c}\text { Number of } \\
\text { channels }\end{array}$ & Parameter & $\begin{array}{c}\text { Measurement } \\
\text { range }\end{array}$ & $\begin{array}{c}\text { Dynamic } \\
\text { range, } \mathrm{Hz}\end{array}$ \\
\hline 3 & $\begin{array}{c}\text { Vibration } \\
\text { acceleration }\end{array}$ & $\begin{array}{c}0-16, \\
2 \%, \mathrm{~mm} / \mathrm{sec}^{2}\end{array}$ & $20-1600$ \\
\hline 3 & $\begin{array}{c}\text { Linear } \\
\text { displacements }\end{array}$ & $0-10,0.05 \%, \mathrm{~mm}$ & $\begin{array}{c}0-250, \\
0.05 \%\end{array}$ \\
\hline 1 & Temperature & $0-540,0.2-5{ }^{\circ} \mathrm{C}$ & - \\
\hline 2 & Power loading & $0-100,0.5 \%, \mathrm{H}$ & $0-350$ \\
\hline 1 & Rotational speed & $\begin{array}{c}0-20,2 \%, \\
\text { rev/min }\end{array}$ & 20 \\
\hline
\end{tabular}

Accelerometer ADXL327 measures 3-axis acceleration in the range from 0 to $19 \mathrm{~m} / \mathrm{s}^{2}$ [1]. Position "7" - Linear sensor LVDT. "Pin" - indenter mounted on a special holder " 4 " with the possibility to move along two axes $X, Y$. Preload of spring is adjusted by screws, $k_{1}$ is the $X$-direction spring stiffness and $k_{2}$ is the $Z$-direction spring stiffness.

Based on the requirements for measurements of dynamic friction parameters on the scheme "Pin on disk" required improvements to the existing measurement system (Table I).

New types of sensors have been applied: loading, linear displacement and acceleration, which increased the accuracy of measuring range and dynamic range for the measurements of dynamic parameters.

Using of these sensors allowed to propose the new research method based on input-output model. A series of tests with the setup showed high efficiency of conducting research on friction and wear.

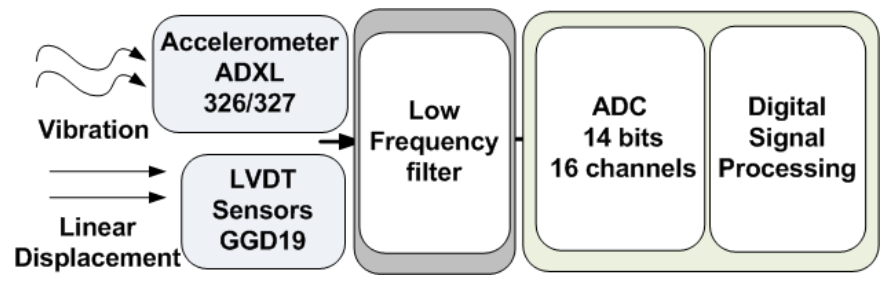

Fig. 3. Schematic diagram of sensor connection. 


\section{MEASUREMENT OF INDENTER'S VIBRATION IN FRICTION CONTACT BY ACCELEROMETER}

There are different types of accelerometers, which are used to determine, detect and control the vibration in mechanisms. With their help such parameters as direction, magnitude, acceleration and others are measured.

One of the world leaders in the manufacturing of microelectromechanical system (MEMS) accelerometers is the Analog Devices company. In its nomenclature accelerometers with one, two and three-axis sensitivity, designed for maximum acceleration from 1.5 to $250 \mathrm{~g}$. Family ADXL - basic unit in which the sensor and electronics are implemented on a single chip.

Two MEMS accelerometer ADXL327 and ADXL326 are used as acceleration sensors (Table II) with different characteristics, since the acceleration test of different materials can vary considerably. Accelerometers are connected to measuring system under a special scheme (Fig. 4). Precision of follower operational real-to-real amplifier AD8554 provides impedance matching at the output of the sensor and the ADC input. Sensor signal is only once passed through an amplifier and gets the digitization, and after this process the digital signal goes through the Fourier transform. In the ADXL327 accelerometer the internal resistor and an external capacitor $\mathrm{C} 1$ form a low-pass filter to cut off the noise above $500 \mathrm{~Hz}$. Accelerometer's and amplifier's supply voltage +3.3 is created by linear voltage regulator LP2950-3.3.

\section{MEASUREMENT OF LiNEAR DisPlaCEMENT}

Linear displacements of the indenter are recorded parallel with measurements of accelerations (constant attached with the disc). The linear encoder has accuracy near $0.25 \%$, the polling frequency to $250 \mathrm{~Hz}$ and a high resolution (up to 10 microns). To match the output of the sensor and turn the ADC voltage range and impedance AD8554 operational amplifier (OA) is used (Fig. 5). To pass signals with frequency higher than $300 \mathrm{~Hz}$ low frequency filters connected with sensors were installed in the circuit.

TABLE II

CHARACTERISTICS OF USED ACCELEROMETERS

\begin{tabular}{|c|c|c|c|}
\hline Parameter & ADXL326 & ADXL327 & ADXL001 \\
\hline Acceleration $(\max ), \pm \mathrm{g}$ & 16 & 2 & 70 \\
\hline Axis & \multicolumn{2}{|c|}{$\mathrm{XYZ}$} & $\mathrm{X}$ \\
\hline Nonlinearity, $\%$ & \multicolumn{2}{|c|}{0.3} & 0.2 \\
\hline Sensitivity $\mathrm{mV} / \mathrm{g}$ & 57 & 420 & 16 \\
\hline The cutoff frequency, $\mathrm{Hz}$ & \multicolumn{2}{|c|}{$X, Y-1600, Z-500$} & 22000 \\
\hline Supply voltage, V & \multicolumn{2}{|c|}{$1.8 \ldots 3.6$} & $3.135 \ldots 6$ \\
\hline Current consumption, mA & \multicolumn{2}{|c|}{350} & 2500 \\
\hline Operating temperature range & \multicolumn{2}{|c|}{$-40 \ldots 85$} & $-40 \ldots 125$ \\
\hline Enclosure Type & \multicolumn{2}{|c|}{ LFCSP-16 } & LCC-8 \\
\hline
\end{tabular}

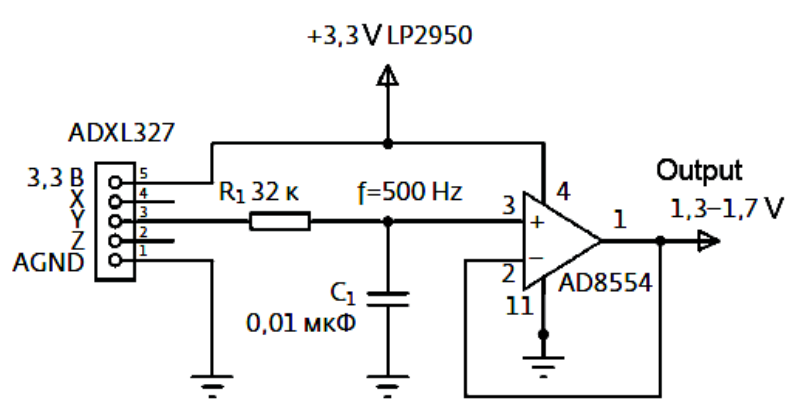

Fig. 4. Functional circuit processing of the analog signal.

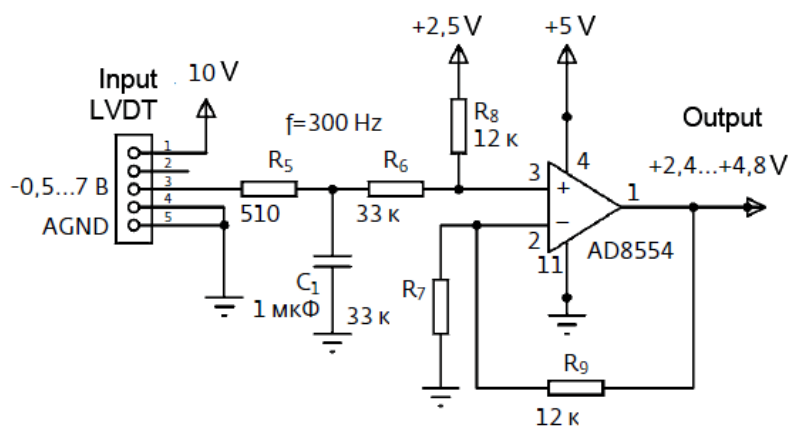

Fig. 5. Functional circuit of analog signal level shifting.

It is impossible to directly implement the connection from the sensor to ADC because of the low output signal, it is necessary to use a differential operational amplifier.

Differential amplifier is an electronic amplifier with two inputs and the output of which is equal to the difference between the input voltage multiplied by variable constant. It is used when there is need to allocate the high-voltage difference of the in-phase components. In-phase component is the component of analog signal present with the same sign, the amplitude and phase of all outputs.

The designed analog measuring system with a set of required functions consists of 5 processing cards and the amplification of signals comes from the sensors specially designed for the study of the dynamics experiments for devices Tribal-3 and the "pin on disc" tests.

For simplicity we are considering only one of the chains connecting the sensors, namely to connect gage. The circuit consists of two OA blocks one of them OP 293, another $8552 \mathrm{AD}$. Objects of the first amplifier are to amplify the signal for 100 times and to make an appropriate choice of resistor. The second $\mathrm{OA}$ is used to level shift the signal in the range from -2 to 2 , is used to convert from 2.4 to $4.8 \mathrm{~V}$ in the sigma-delta ADCs in digital 14 bit signal.

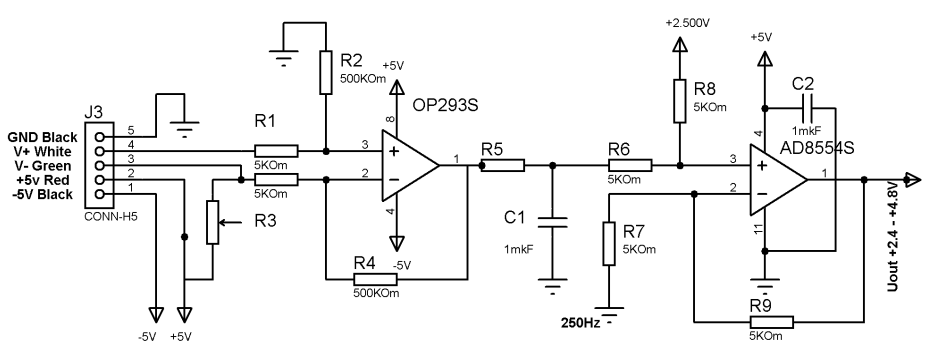

Fig. 6. Schematic diagram of an electronic load cell EX601D connected to a data acquisition system. 
Consider in more detail the connection diagram of the strain gauge and analog board on this basis. This circuit consists of two parts, the right part represents the diagram of the OA to bias at the ADC input signal discussed in the previous chapter. The left diagram is a differential signal amplifier. Calculation of the output voltage provides according to the following formulas:

$$
\begin{gathered}
U_{\text {out }}=U_{3} k_{3}-U_{2} k_{2}, \\
k_{3}=\frac{\left(R_{4}+R_{3}\right) R_{2}}{\left(R_{2}+R_{1}\right) R_{3}} \text { and } k_{1}=\frac{R_{4}}{R_{3}} .
\end{gathered}
$$

If $R_{4}=R_{2}$ and $R_{1}=R_{3}$

$$
U_{\text {out }}=\frac{R_{4}}{R_{3}}\left(U_{3}-U_{2}\right) .
$$

Resistance ratio is selected to be 1 in 100 to provide amplification of the input signal 100. Also amplified noise with a strain gauge, so hereinafter referred to as a lowpass filter is provided to $250 \mathrm{~Hz}$ and its parameters are selected experimentally depending on the frequency of change of the mechanical impact on the sensor.

Schematic diagram of the program is executed through design Proteus 7.0. Perform layout boards in such programs is very convenient, you can create all of the printed circuit board (PCB) layout, select components (with specific types of buildings), the trace mask for the speedy creation of manufacturing .

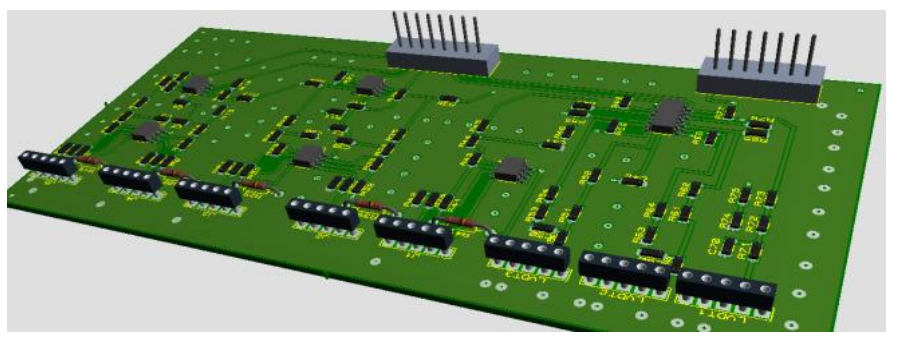

Fig. 7. The figure shows the $3 \mathrm{D}$ images prepared for the manufacture of printed circuit boards preamp and analog-to-digital converter.

\section{INVESTIGATION OF NEW ROUGHNESS PARAMETER}

New method of measuring surface roughness was investigated. Usually for technical surfaces produced a comprehensive inspection of selected properties, such as shape, waviness and roughness is done.

The surface roughness has a basic influence on the functional properties of friction pairs in dry and mixed conditions. Generally in measurement profilometer is used because of its simplicity and accuracy.

In this work profiler Hommel Tester T8000 "Hommelwerke, GmbH" company (Switzerland), was used. The basic length of measurements was possible depending on sample length of 1 to $10 \mathrm{~mm}$ at $0.15 \mathrm{~mm} / \mathrm{s}$.

In practice, two or five parameters are required for the full surface description, obtained after the statistical analysis the profile. Was applied of wavelet decomposition is onedimensional signal $x(t)$ to the basis constituted by shifts and various scale copies the prototype function (mother wavelet function $\psi(t))$ [3].
The analyzed profile from discrete wavelet decomposition is decomposed into the sum of the components of the smoothed last level (A6) and details of all levels (D6...D1). Daubechies wavelet $d b 4$ with central frequency $f_{r}=0.7143 \mathrm{~Hz}$ was used as a mother. The frequency corresponding to each level of decomposition was calculated using the equation:

$$
F r_{j}=\frac{f_{r} f_{s}}{2^{j}}
$$

where

$j=0, \ldots, 6 \quad$ order of decomposition;

$f_{s} \quad$ sampling frequency, $\mathrm{Hz}$, which usually can be defined before experiment.

Fig. 6 presents a two-level decomposition, where each level received the reconstructed signals.

For obtaining signal components for analysis typically is used fast Fourier transform algorithm, followed by estimation of the spectral energy of these components. The spectral energy density of the signal $E(f)$ is equal to the square of the Fourier transform signal [4]:

$$
E(f)=|X(f)|^{2}=\left|\int x(t) e^{-2 \pi i f t} d t\right|^{2},
$$

where $X(f)$ is the signal spectrum, which describes the distribution of signal energy over frequency.

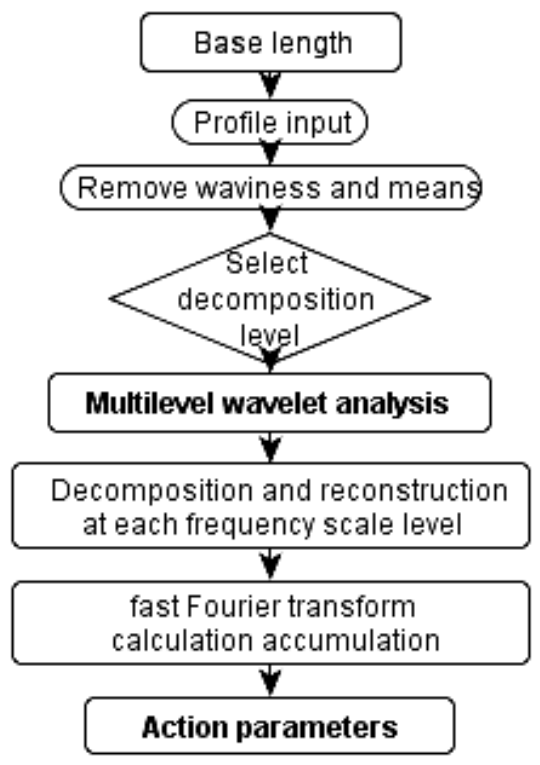

Fig. 8. Scheme of algorithm determines the action parameters of the surface.

The accumulation of spectral energy called full signal energy within the frequency band $\left(f_{1}\right.$ to $\left.f_{2}\right)$ is defined by the integral:

$$
C=\int_{f_{1}}^{f_{2}} E(f) d f .
$$

The stored energy (full) defines the numerical value of the energy distributed across the spectrum. 


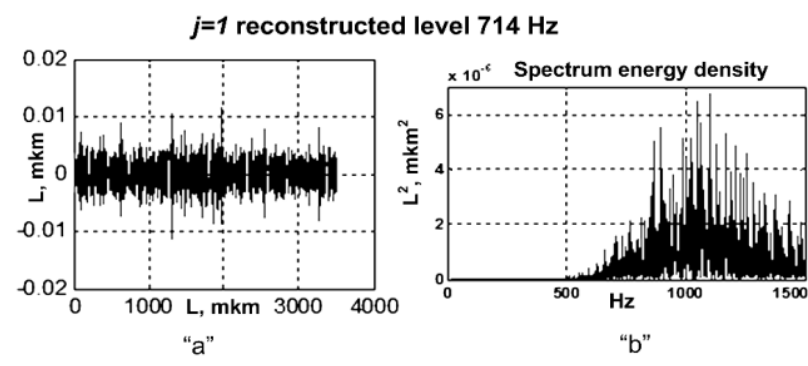

Fig. 9. "a" - reconstructed signal ; "b" its power spectral density.

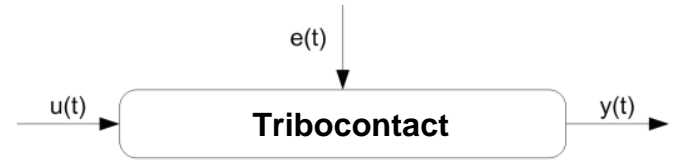

Fig. 10. The input-output model of a friction dynamic system.

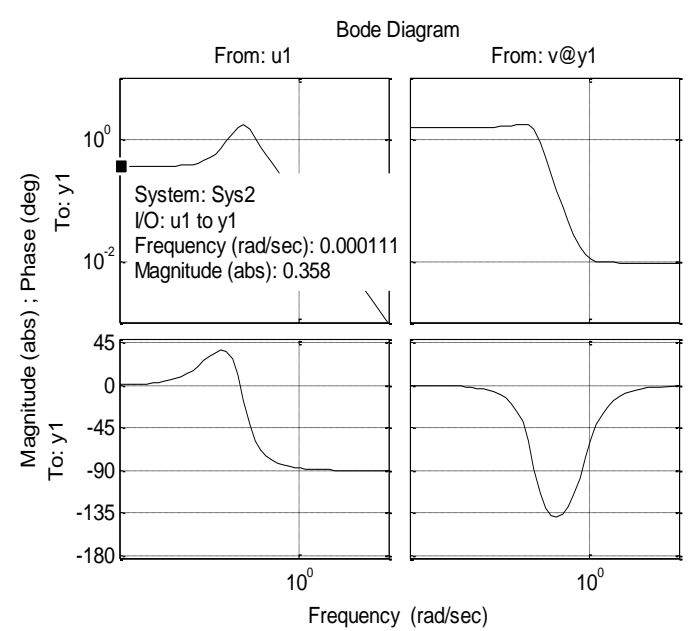

Fig. 11. Typical amplitude frequency response (Bode diagram).

Action parameter - the integral value, which determines the ratio of stored energy $C$ with a frequency range that is happening in this accumulation $f_{1}-f_{2}$, for each frequency scale level

$$
h_{1}=\frac{C}{\left(f_{2}-f_{1}\right) f_{\max }} \text { and } h_{2}=\frac{E \max }{f_{\max }},
$$

Where: $E$ max is the maximum value of the spectral energy density, $f_{\max }$ is the maximum frequency for the selected interval and $C$ is limit value (accumulated) energy storage.

Action parameter $h_{1}(\mathrm{~J} \cdot \mathrm{s})$ determines the relation between the level of maximum accumulation of the signal energy, related to the frequency corresponding to the maximum value of the spectral energy density, and frequency interval in which energy is accumulated. Parameter $h_{2}(\mathrm{~J} \cdot \mathrm{s})$ describes the ratio of the maximum spectral density to the appropriate frequency value.

The author obtained a new parameter of the transfer between the interacting surfaces, developed the theory of the transition from the mechanical action to the action of the frequency for the task of tribological measurements. Used to achieve the wavelet and spectral analysis in one integrated parameter describing the properties of the surface. Parameter allows you to analyze the energy transfer processes in the frequency scale levels.

\section{INPUT-OUTPUT ALGORITHM}

Tribological system can be considered from the inputoutput point of view (Fig. 10). Input dynamic parameters of the tribological system are: sliding speed, rolling speed and contact pressure. Heat parameters are the temperature, heat flow and a temperature gradient. Output parameters of the tribological system are the impact of system on the environment and the system response to external influence [6], [7], [8].

The input signals $u$, the random impact $e$, the output parameters $y$ influenced the object of studies. The signals $u, e$, $y$ are vectors of any dimension and function of time such as $u(t)$.

Experimental data is discrete signal value. The problem of identification is in finding dependencies between input and output signals [4]. The diagram (Fig. 11) has an agreed measurement data input and output actions, which were measured in the same range.

In the general case "input" and "output" of the system can be described by a differential equation of $n$ order [6], [7], where the right-hand side is the "input" and the parameters $a_{0}$, $a_{1}, \ldots a_{n}$, are selected based on a specified an input signal, $b_{0}$, $b_{1}, \ldots b_{n}$, assumed to be given [6], [7].

$$
\begin{aligned}
& a_{0} x^{(n)}+a_{1} x^{(n-1)}+\ldots+a_{n} x= \\
& =b_{0} y^{(m)}+b_{1} y^{(m-1)}+\ldots+b_{m} y .
\end{aligned}
$$

The differential equation of motion of the second order with $n=2$ satisfies the system model based on the study of equipment "Tribal-T" in which there is relative movement of the two surfaces. The relative movement in the dynamics of mechanical systems can be presented by Newton equation [6]. Simulation of tribological systems is performed according to the known dynamic model of second-order system [1]:

$$
\frac{d^{2} x}{d t^{2}}+2 \zeta \frac{d x}{d t}+\omega_{0}^{2} x=f(t),
$$

where $\zeta$ is damping factor, $(1 / \mathrm{s}), f(t)$ is external influence on system, and $\omega_{0}$ is natural frequency, $\mathrm{Hz}$.

The dynamic properties of the system are determined by solving the differential equation in the Matlab [2], further the transfer function parameters located Autoregressive moving average model, for example, (Fig. 9) is plot of amplitudefrequency response [5].

Discrete second-order model, equivalent

Sys $=\operatorname{canstart}(z, 2,1,[0,1,0])$,

where 2 is order of differential equation, 1 is the number of system inputs, 0 is accounting delay (the delay counted), 1 is accounting interference (noise is accounting), 0 is the initial state (zero initial conditions).

The program under Matlab was written for the numerical calculation of the tribological parameters, it allows to process data immediately after the experiment.

Changes in the surface roughness of the metal samples were not detected, the surface of PTFE wore in the first five minutes, taking the irregularities form of metal specimen. 
TABLE III

CHARACTERISTICS OF USED EQUIPMENT

\begin{tabular}{|c|c|c|}
\hline System & $\begin{array}{c}\text { Damping } \\
\text { factor, } \zeta,(1 / \mathrm{s})\end{array}$ & $\begin{array}{c}\text { Dynamic } \\
\text { friction coefficient, } K d\end{array}$ \\
\hline Tribal-T & $0.7 \pm 0.017$ & $0.2770 \pm 0.071$ \\
\hline Pin on disc & $0.6903 \pm 0.029$ & $0.2855 \pm 0.0186$ \\
\hline
\end{tabular}

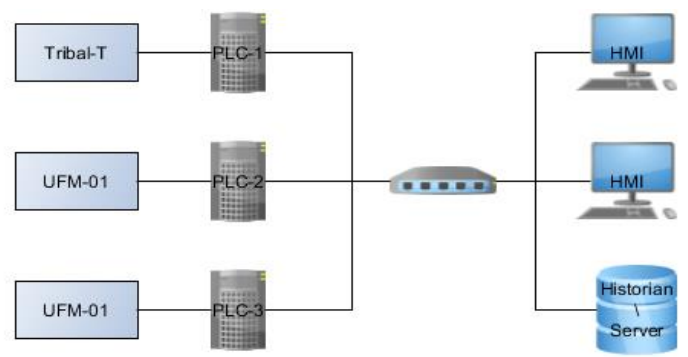

Fig. 12. Laboratory system for data acquisition and control.

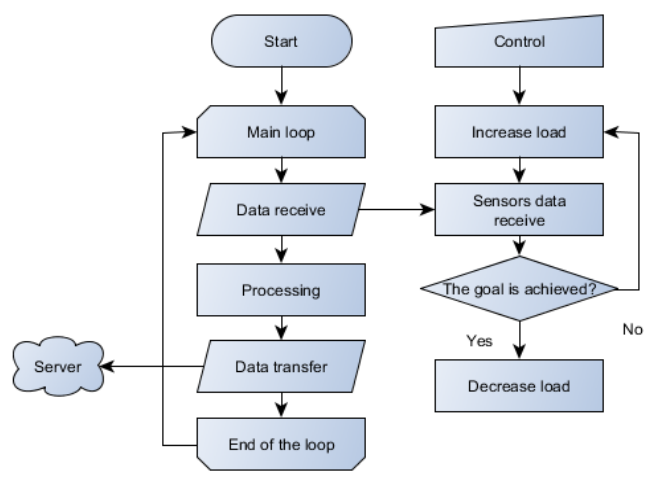

Fig. 13. PLCs program algorithm.

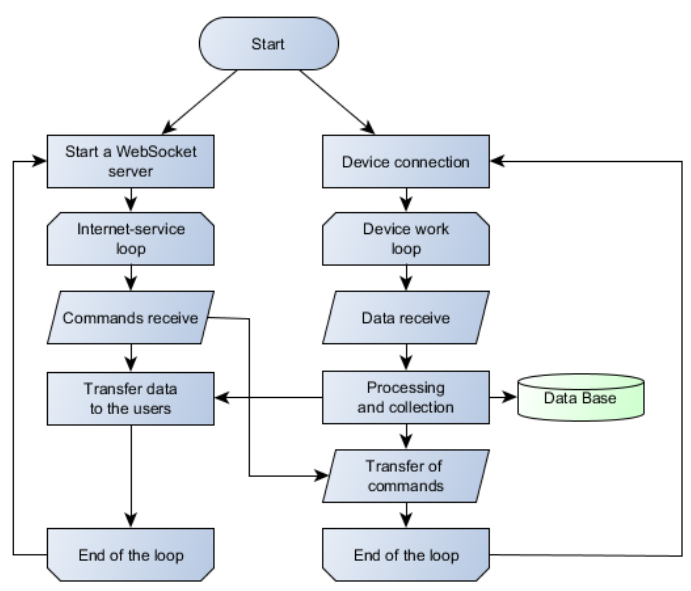

Fig. 14. Server's program algorithm.

From the measured acceleration values a slight increase of roughness PTFE in the initial test period can be concluded. The damping coefficient is practically the same for all possible tests due to the unique properties of PTFE - self-lubricity and viscosity [2].

In various materials there are various stability factors of the system. Mathematical model of the device allows to set the desired motion law in a closed-loop system. Task of regulation is not considered. In this paper we only used the methods of control theory to analyze the frictional quality.

\section{DATA COLLECTION AND CONTROL}

Data retrieval and monitoring of device status is one of the engineering problems. Selecting solutions to this problem depends on the nature and specifics of the laboratory. We have tested the system with different architecture. Each coping with the main task of obtaining information from the device but having its own peculiarities. As a result, the laboratory system for data acquisition and control was developed, bringing together all the measuring equipment. It is a customizable and scalable SCADA-system that combines control and data acquisition not only with "Tribal $-\mathrm{T}$ ", but with a universal friction machine (MTU- 01 and modified MTU- 01), designed for "Pin on disc" tests. (Fig. 12)

There are two modes of operation: design mode and run mode (runtime). These modes are not necessarily mutually exclusive: you can edit a project on one computer, engineer and download it, and it will be updated on the user's machines. Graphical interface is created in SCADA, data sources are configured with field devices, it is responsible for the interaction of the user (operator, manager, technologist) with what is happening in the workplace, as well as the archiving of all relevant data in the database.

A SCADA system consists of the following subsystems:

Control devices. Human-Machine Interface (HMI) and server with databases, logs, and other information

Remote devices. Laboratory equipment ("Tribal-T", MTU01), programmable logic controllers (PLC), ADC, etc.

In this system, HMI is a program located on the server and running on remote computers. HMI provides the control of laboratory machines and viewing of data obtained during the study. Compared with the previous generation system, we can manage the entire laboratory from a single computer connected to a laboratory network. A server with databases stores information about all the research and provides access to it.

PLC's program provides data transfer to the server and receives instructions from it. The program created for PLC is universal - it allows you to choose, using the HMI, control channels and data transmission. This makes the whole system scalable - the acquisition of the new equipment requires only connecting it to the new PLC and opening it in the HMI.

The PLC's program's objective is to collect data, shape them into packets and send them to the server. The following figure shows a block diagram of the program. (Fig. 13)

The launching of the device has started. After starting the infinite loop it begins checking for new commands and data (receive data). When the command specifying the mode of operationis given, the program keeps track of motors in accordance with this mode (engine control unit). When data is received from the $\mathrm{ADC}$, the program generates one package and sends to the server (sending data).

The purpose of the server's program is to organize the device management and to monitor the data. The following figure shows a block diagram of the program. (Fig. 14).

The Server Software runs in two streams. Each stream runs its loop. The first loop provides the WebSocket-server, sending data to each client is connected. Upon receiving control commands, they are processed and go to the device in 
the loop with the device. The second cycle data received from the device, is stored and transmited in a first cycle for publication. Unfortunately, the technologies that built modern SCADA-systems focused primarily on the control of problem solving process. Security Features are either absent or implemented as residual. Therefore, the system will be further developed. Currently, the issue of security is easily solved. The control is on a local network, but it is often inconvenient.

\section{CONCLUSION}

New hardware and software tools were developed that allow high-precision register tribology parameters in the real time, at basis of measuring equipment "Tribal-T" and "Pin on disk".

New tribology parameter was proposed - the "action parameter" that allows to analyze the processes of energy transfer at different frequency scale levels, through the use of wavelet and spectral analysis. The authors created the method of calculating the transfer of action in tribocontact as an integral characteristic, defined at each frequency level.

The research approaches applied in the equipment "Pin on disk" were similar to those used in input-output systems, with additional measurement channels by which the dynamics of friction processes and wear in real time.

\section{REFERENCES}

[1] A. Tyurin, I. Kalapishina and M. Viugin, "Accelerometer Analog Devices - investigation of vibration induced friction," Electronics STB technical-science J., 4, 2013. pp. 116-120.

[2] A. Tyurin and G. Ismailov, "Vibration monitoring auto autowave process in tribocontact Polytetrafluoroethylene-metal," Constraction of composite materials, no. 2, 2013, pp. 58-64.

[3] V. Musalimov, O. Dik and A. Tyurin, "Energy parameter of discrete wavelet transform: an application to tribological and physiological signals," Scientific-technical. J. of Information Technologies, Mechanics and Optics, 2009, pp. 27-34.

[4] V. Musalimov, O. Dik and A. Tyurin, "Action parameter energy spectrum of veivlet transform," RUIFMO Instrument making, 2009. no. 5. s. $10-11$.

[5] V. Musalimov, F. Petzold and A. Tyurin, "Messung und Bewertung des Energiezustands von Werkstoffoberflächen," Workshop "Flexible Montage” proc., Technische Universität Ilmenau, 2010, pp. 8-15.

[6] V. Musalimov, M. Larin and A. Tyurin, "Using Optical Tracking Technology to Estimate Cyclical Linear Motion," 13th Int. Symp. "Topical problems in the field of electrical and power engineering" and "Doctoral School of Energy and Geotechnology II", Parnu 2013, pp. 319-322.

[7] V. Musalimov and V.A. Valetov, "The dynamics of frictional interaction," St. Petersburg State University of Information Technologies, 2006, pp. 100-110.

[8] G. Goossens et al., "Embedded software in real-time signal processing systems: Design technology," Proc. IEEE, vol. 85, issue 3, 1997, pp. 436-455. http://dx.doi.org/10.1109/5.558718

[9] P. Horowitz and W. Hill, The Art of Electronics, 2nd ed., Cambridge University Press, 1989, ISBN 978-0-521-37095-0.

[10] J. Dostál, Operational amplifiers, Butterworth-Heinemann, 1993, 387 p.

[11] R. Tokheim, Digital Electronics: Principles and Applications.

[12] "Electrical Science", DOE Fundamentals Handbook, 1998, vol. 1-4.

[13] P. Green, "Usability analysis of visual programming environments: A 'cognitive dimensions' framework," J. of Visual Languages and Computing, vol. 7 1996, pp. 131-174. http://dx.doi.org/10.1006/jvlc.1996.0009

[14] Some electronics circuit design and microcontrollers [Online]. Available: http://easyelectronics.ru/

[15] S. M. Kazraji, R. B. Soflayi and M. B. B. Sharifian, "Sliding-Mode Observer for Speed and Position Sensor-less Control of Linear-PMSM," The Scientific J. of Riga Technical University. Electrical, Control and Communication Engineering. vol. 5, 2014, pp. 20-26. http://dx.doi.org/10.2478/ecce-2014-0003
[16] The WebSocket API, W3C Working Draft specification of the API.

[17] The WebSocket protocol, Proposed Standard published by the IETF HyBi Working Group.

[18] How Web Sockets Interact With Proxy Servers, [Online]. Available: http://www.infoq.com/articles/Web-Sockets-Proxy-Servers. Retrieved: 2011-12-10.

[19] IANA Uniform Resource Identifer (URI) Schemes, [Online]. Available: http://www.iana.org/assignments/uri-schemes/uri-schemes.xhtml. Retrieved: 2011-11-14.

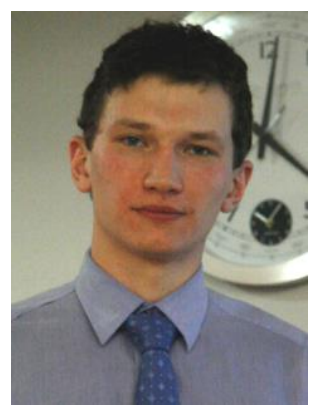

Andrey Tyurin is a researcher with the Department of Mechatronics, St. Petersburg National Research University of Information Technologies, Mechanics and Optics, Russia. His research interests include: tribological properties of new construction materials and coatings, testing of lubricants, experimenting and modelling, development and adaptation of modern methods and techniques for the friction and wear investigations, development of software for the evaluation of friction parameters and surface roughness profiles. He has participaed at a number of international conferences. He has been on Euler Master internship at Technische Universität Ilmenau, Germany, where he worked on diffusion welding of materials, investigation on surface properties at different treatment methods and its influence on the welding process

Address: ITMO University, Kronverkskiy Pr. 49, Saint-Petersburg, 197101, Russia. E-mail: a.e.tyurin@gmail.com

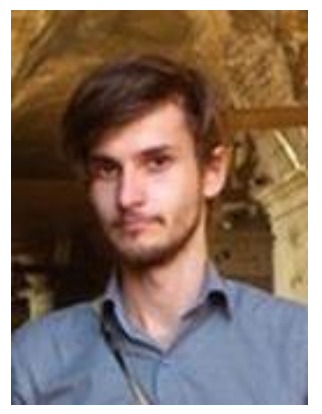

German Ganus holds a Master degree and is with the Department of Mechatronics, St. Petersburg National Research University of Information Technologies, Mechanics and Optics, Russia. His research interests include electroactive polymer materials and coatings; testing of actuators, experimenting and modelling. He is a developer of software, WEB and mobile application and is researching swarm robotics.

Address: ITMO University, Kronverkskiy Pr. 49, Saint-Petersburg, 197101, Russia.

E-mail: germanganus@gmail.com

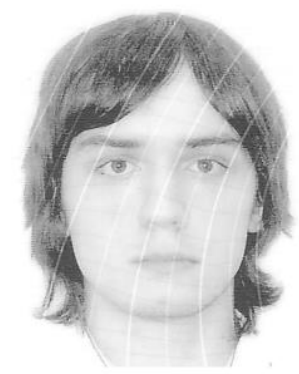

Mikhail Stepanov is a postgraduate student with the Department of Mechatronics, St. Petersburg National Research University of Information Technologies, Mechanics and Optics, Russia. He is researching friction interaction of solids taking into account the elastic, thermal and acoustic vibrations occurring in the contact zone.

Address: ITMO University, Kronverkskiy Pr. 49, Saint-Petersburg, 197101, Russia.

E-mail:stmivi@rambler.ru

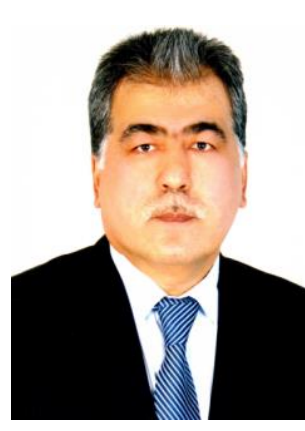

Gafurzhan Ismailov holds a Ph.D. He is an Associate Professor with the Department of Applied Mechanics, Tomsk State Pedagogical University. He studied Mechanical Engineering, specialty "Building and road machines." His research is in the field of dynamics and machine durability, tribology and mechanic cable. $\mathrm{He}$ is the author and developer of devices and methods for the study of tribological properties of materials. He is investigating friction contact forces and conducting experimental studies to determine the mechanical and tribological properties in cable elements, with the aim to

create heavy-duty cable coatings.

Address: Tomsk State Pedagogical University, Kievskaya Str., 60, Tomsk, 634041, Russia.

E-mail: gmismailov@ rambler.ru 